\title{
Polymorphonuclear neutrophil infiltration into ischemic infarctions: myth or truth?
}

\author{
Hannu Kalimo • Gregory J. del Zoppo • \\ Anders Paetau $\cdot$ Perttu J. Lindsberg
}

Published online: 16 February 2013

(C) Springer-Verlag Berlin Heidelberg 2013

Knowledge builds on steps considered scientific truths, which shall endure the test of time and variations in circumstances. For decades, neuropathologists have used the sequence of cellular changes including immune cell reaction in infarcted brain tissue for timing the development of the infarction [1, 13]. A generally accepted concept has been that polymorphonuclear neutrophils (PMN) can cause plugging of microvessels within the vascular territory of an occluded artery even when reperfused [2], and they extravasate and invade the brain parenchyma at early stages of infarction (within 15-24 h; e.g., [1, 10, 11, 13, 15,

H. Kalimo $(\bowtie) \cdot$ A. Paetau

Department of Pathology, Haartman Institute,

University of Helsinki, P.O. Box 21 (Haartmaninkatu 3),

00014 Helsinki, Finland

e-mail: hannu.kalimo@helsinki.fi

A. Paetau

e-mail: anders.paetau@helsinki.fi

H. Kalimo

Department of Biomedicine/Forensic Medicine, University

of Turku, 20520 Turku, Finland

\section{G. J. del Zoppo}

Department of Medicine (Division of Hematology) and the Department of Neurology, University of Washington,

Seattle, WA 98104, USA

e-mail: grgdlzop@u.washington.edu

P. J. Lindsberg

Department of Neurology, Helsinki University Central Hospital,

Helsinki, Finland

e-mail: perttu.lindsberg@hus.fi

P. J. Lindsberg

Department of Clinical Neuroscience, Molecular Neurology

Research Programs Unit, University of Helsinki,

Helsinki, Finland
17]. The infiltration of PMNs into ischemic infarcts has led to the hypothesis that besides adhering to the endothelium and plugging microvessels before extravasation (no-reflow phenomenon), PMNs release a palette of neurotoxic substances, which contribute to neuronal cell death as a component of ischemia-reperfusion injury. Having been confirmed in many experimental brain ischemia studies, this hypothesis has stimulated trials of therapeutic interventions based on combatting this PMN response by various means (e.g., $[5,14])$. Such therapeutic trials targeting PMNs have given inconsistent results in both experimental studies and clinical trials.

This has prompted Drs. Engelhardt and Sorokin to recruit-as the authors state it-a multicenter team comprising stroke researchers, neuropathologists and basic scientists for collaborative investigation to determine the temporo-spatial relationship between immune cells and blood vessel microarchitecture in the mouse and human brain at early (acute) stages after ischemia. The authors have applied a wide arsenal of elaborate methods. Transient focal ischemia was induced in mice by occluding the middle cerebral artery for 30-90 min with a microfilament followed by recirculation from $6 \mathrm{~h}$ up to 2 weeks. Comparison with human cases was performed by assessing brain histopathology in 25 human victims of stroke. In addition, under in vitro flow conditions, they have tested PMN migration across the endothelial monolayer exposed to oxygen and glucose deprivation.

The results of the study of Enzmann et al. [6] are challenging to neuropathologists, who for decades have observed PMN infiltration and interpreted it as an inherent component in the sequence of events in infarct maturation. Using specific markers for inflammatory cells and for structures of the neurovascular unit, Enzmann et al. put forth that in their experimental ischemia model PMNs 
accumulate on the luminal surfaces or in the perivascular spaces of cerebral vessels, mainly arterioles, whereas their spread to the parenchyma is efficiently restricted by the glia limitans (parenchymal basement membrane on the astrocytic endfeet). They arrived at the same conclusion in reviewing brains of 25 human victims of stroke (with hemorrhagic infarcts excluded). Furthermore, they suggest that the majority of the PMN-looking cells in infarcted parenchyma are in fact apoptotic cell bodies (their cell of origin is not proposed), which are erroneously regarded as PMNs. However, it is known that PMNs also succumb by apoptosis [9]. This discrepancy compared to previous experimental and clinico-pathological interpretations and reports requires closer scrutiny. Because the authors are experienced researchers and their methods appear reliable and sophisticated, there must be an explanation for this discrepancy, which could relieve neuropathologists and other researchers of blushing from embarrassment.

It is difficult to believe that PMNs can be seen in acute brain infarcts only by old-fashioned neuropathologists or that neuropathologists have for decades misinterpreted the cells, which they have identified as PMNs on the basis of morphology and distribution. There is a vast literature on PMN efflux in ischemic brain infarctions, which cannot be reiterated here-just as examples, the cluster of cells in Fig. 3.71 in Greenfield's textbook [7] depicting an infarct on day 2 or the cells in our Figs. 1 and 2 showing acute infarcts on day 2. In a classic study, Garcia et al. [10] used electron microscopy to identify PMNs in brain parenchyma and found them to peak at $24 \mathrm{~h}$ in anesthetized rats. Lindsberg et al. [12] applied the same immunomarkers, CD15 for PMNs and ICAM-1 for PMN adhesion to activated endothelial cells, as Enzmann et al. in human stroke cases. They observed an up to 25 -fold increase in the number of PMNs as early as $15 \mathrm{~h}$ after the insult, which further increased several fold during the next 2 days. At the earliest stages, PMNs were not exclusively, though predominantly, localized intraluminally and perivascularly, but definitely also intraparenchymally (Fig. 2). Furthermore, distribution of PMNs spatially and temporally corresponded to the markedly increased number of microvessels with strong ICAM-1 staining. In the less acute stages, ghosts of PMNs as well as their phagocytosis by invading macrophages were found in cortical and subcortical regions, outside vessel lumina or perivascular spaces, and distant to leptomeninges or hemorrhagic foci.

It is obvious that in routine diagnostic work neuropathologists have hardly used immunomarkers for PMNs or basal lamina to pinpoint the location of PMN-looking cells. However, neuropathologists have certainly been capable of identifying perivascular PMNs, even if they may have interpreted them to be intraparenchymal. Thus, the results of Enzmann et al., though challenging, do not actually

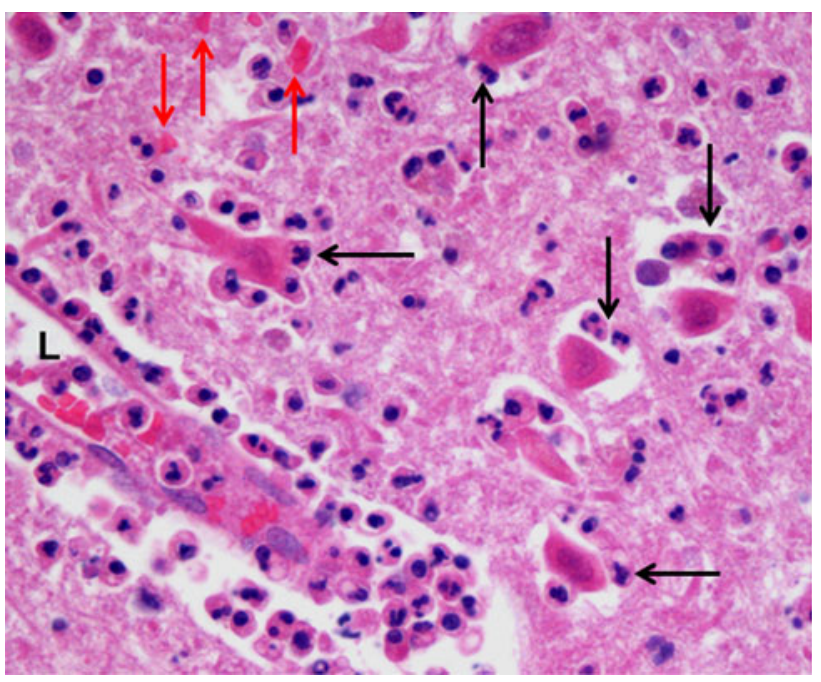

Fig. 1 An acute infarct in hippocampal CA4 sector on day 2. Numerous PNMs are visible within the vascular lumen $(L)$, in the perivascular space as well as diffusely in the parenchyma, some appearing to attack ischemically injured eosinophilic neurons (five marked with black arrows). This infarct is incipiently hemorrhagic, as verified by small clusters of erythrocytes (red arrows), indicating disruption of the glia limitans. Note that hemorrhage is only minimal (H\&E)

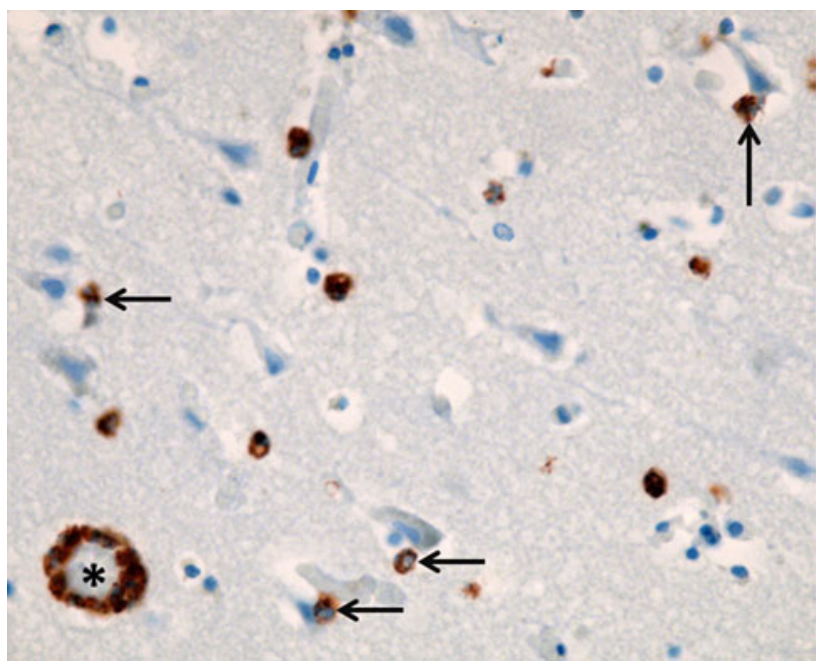

Fig. 2 Another acute infarct in the frontal cortex on day 2. As in Fig. 1, PMNs, immunostained with myeloperoxidase antibody, are seen in the perivascular space (asterisk) and diffusely in the parenchyma. Again, some PMNs appear to attack ischemically damaged, shrunken neurons (four marked with arrows). There are no definite erythrocytes visible, though elsewhere at a distance from this site some lesser hemorrhages were observed (anti-myeloperoxidase and hematoxylin counterstain)

compromise neuropathologists' routine practice to use the appearance of PNMs for infarct timing, since - similarly as in many previous studies - in mice of this study PMNs peaked at 18-24 h, and they were also present in the acute stage of human stroke, though mainly in perivascular spaces. 
The results of Enzmann et al. demonstrate that the parenchymal basement membrane (glia limitans) is a tough barrier appearing to prevent the spread of PMNs into the parenchyma in early stages of ischemia. Enzmann et al. detected intraparenchymal PMNs most often in regions with more severe tissue damage (tissue tears). The authors suggest that vascular disruption might be a very logical explanation for the detection of PMNs in the CNS parenchyma in hemorrhagic stroke cases, i.e., glia limitans should be broken before the ischemic attraction induces major extravasation of PMNs to the parenchyma. In this study, the authors did not include/examine mice or patients with incipiently or full-blown hemorrhagic infarcts. Later they indicated that they do observe unequivocal extravasation of PMNs in hemorrhagic infarcts (personal communication). It is not known whether glia limitans disruption of such a severity that it allows active transmigration of PMNs would also allow extravasation of erythrocytes, i.e., make the infarct hemorrhagic. It must also be kept in mind that in human cerebral infarctions, the vascular disruption and inflammatory response can be quite patchy and the phase of prevalent PMN infiltration is in fact a transient phenomenon, so it may be missed, if the brains are not systematically sampled to capture regions with different degrees of neuronal damage. Yet, it is somewhat unexpected that in all 25 of the authors' stroke patients, the vascular lesions had been so mild that breakdown of the glia limitans and consequent extravasation of PMNs had not occurred. Possibly the authors' very careful exclusion of even minimally hemorrhagic stroke cases resulted in a cohort with few intraparenchymal PMNs.

The authors bravely suggest that their results are one explanation why most of the clinical trials targeting PMNs to minimize infarct volume have not been successful. The suggested efficiency of the glia limitans as a barrier cannot be excluded as a potential contributor to unsatisfactory results in clinical trials targeting PMNs. The toxic effect of PMNs from beyond the glia limitans on neurons supplied by those microvessels would be expected to be relatively weak. A major effect would require closer contact, similar to that reported by Dr. Sorokin's team (refs. 1 and 88 in Enzmann et al.) in experimental encephalomyelitis, where induction of symptoms requires immune cell penetration of the glia limitans into the CNS parenchyma. Here, inhibition of any effect by anti-PMN treatments would be expected to have a minor effect. It is, however, also possible that PMNs could act on neurons from a distance, in a fashion compatible with the concept of the neurovascular unit [3, 4]. One simply cannot be sure. In our Figs. 1 and 2 on acute infarcts, intraparenchymal PMNs appear next to (and attacking?) neurons undergoing ischemic damage. Alternatively, they are acting to eliminate dying cell bodies as debris.
This raises several questions: (1) after what kind of and how severe an ischemic injury does the glia limitans break down? (2) Where do these events occur (are events in the cortex different from those in the striatum)? (3) Are the observations in rodents translatable to humans? (4) Are those PMNs that have extravasated in hemorrhagic (incipient or full blown) infarcts "deadly toxic" to ischemically injured neurons and does such an attack call for some therapeutic intervention? Thus, the pathobiology of the breakdown of the glia limitans in ischemia, e.g., the role of matrix metalloproteases in this process [4], deserves further studies, as does the direct toxic effect of PMNs on individual ischemic neurons.

We agree with the authors' skepticism that the exact roles of PMNs in ischemic stroke remain unclear and need further investigation. But we are perhaps even more skeptical, since we claim that most likely the hypothesis of antagonizing PMN activity in human stroke has never been adequately put to the test: none of the trials have provided definite proof of the purported nominal effect, i.e., the inhibition of the PMN response [3]. For example, enlimomab (multiple doses of murine anti ICAM-1 antibody tested in an unfortunate clinical stroke trial), instead of antagonizing PMNs and inflammation, in fact activated PMNs and the endothelium in multiple parallel ways $[8,16]$. This is in line with the aggravating effect of PMNs in acute stroke. Clinical intervention targeting PMNs needs careful monitoring of the tested hypothesis before the hypothesis can be abandoned.

The report by Enzmann et al. is thought-provoking. It will raise considerable interest as well as objections, since it challenges old established observations-invasion of PMNs to acute ischemic lesions and their toxic effect on ischemic neurons. The presence or absence of these phenomena should have logical explanations that should be searched for in further studies. Fortunately, at least for the time being, neuropathologists can continue to use the appearance of PMNs (perivascular or intraparenchymal) as a well-established landmark in the morphological staging of the evolution of cerebral infarcts in their daily practice.

\section{References}

1. Chuaqui R, Tapia J (1993) Histologic assessment of the age of recent brain infarcts in man. $J$ Neuropathol Exp Neurol 52(5):481-489

2. del Zoppo GJ, Schmid-Schönbein GW, Mori E, Copeland BR, Chang CM (1991) Polymorphonuclear leukocytes occlude capillaries following middle cerebral artery occlusion and reperfusion in baboons. Stroke 22(10):1276-1283

3. del Zoppo GJ (2004) Chapter 9: Lessons from stroke trials using anti-inflammatory approaches that have failed. In: Dirnagl U, Elger B (eds) Neuroinflammation in stroke. Springer, Berlin, pp 155-184 
4. del Zoppo GJ (2013) Toward the neurovascular unit a journey in clinical translation: 2012 Thomas Willis lecture. Stroke 44(1): 263-269

5. Emerich DF, Dean RL 3rd, Bartus RT (2002) The role of leukocytes following cerebral ischemia: pathogenic variable or bystander reaction to emerging infarct? Exp Neurol 173:168-181

6. Enzmann G, Mysiorek C, Gorina R, Cheng YJ, Ghavampour S et al (2013) The neurovascular unit as a selective barrier to polymorphonuclear granulocyte (PMN) infiltration into the brain after ischemic injury. Acta Neuropathol. doi:10.1007/s00401012-1076-3 (Epub ahead of print 2012 Dec 27)

7. Ferrer I, Kaste M, Kalimo H (2008) Vascular diseases. In: Love S, Louis DN, Ellison DW (eds) Greenfield's neuropathology, 8th edn. Hodder Arnold, London, pp 121-240

8. Furuya K, Takeda H, Azhar S, McCarron RM, Chen Y et al (2001) Examination of several potential mechanisms for the negative outcome in a clinical stroke trial of enlimomab, a murine anti-human intercellular adhesion molecule-1 antibody: a bedside-to-bench study. Stroke 32(11):2665-2674

9. Gabelloni ML, Trevani AS, Sabatté J, Geffner J (2013) Mechanisms regulating neutrophil survival and cell death. Semin Immunopathol (Epub ahead of print 2013 Feb 1)]

10. Garcia JH, Liu KF, Yoshida Y, Lian J, Chen S, del Zoppo GJ (1994) Influx of leukocytes and platelets in an evolving brain infarct (Wistar rat). Am J Pathol 144(1):188-199
11. Hallenbeck JM, Dutka AJ, Tanishima T, Kochanek PM, Kumaroo KK et al (1986) Polymorphonuclear leukocyte accumulation in brain regions with low blood flow during the early postischemic period. Stroke 17(2):246-253

12. Lindsberg PJ, Carpén O, Paetau A, Karjalainen-Lindsberg ML, Kaste M (1996) Endothelial ICAM-1 expression associated with inflammatory cell response in human ischemic stroke. Circulation 94(5):939-945

13. Mena H, Cadavid D, Rushing EJ (2004) Human cerebral infarct: a proposed histopathologic classification based on 137 cases. Acta Neuropathol 108:524-530

14. Mori E, del Zoppo GJ, Chambers JD, Copeland BR, Arfors KE (1992) Inhibition of polymorphonuclear leukocyte adherence suppresses no-reflow after focal cerebral ischemia in baboons. Stroke 23(5):712-718

15. Pozzilli C, Lenzi GL, Argentino C, Carolei A, Rasura M et al (1985) Imaging of leukocytic infiltration in human cerebral infarcts. Stroke 16(2):251-255

16. Vuorte J, Lindsberg PJ, Kaste M, Meri S, Jansson SE et al (1999) Anti-ICAM-1 monoclonal antibody R6.5 (Enlimomab) promotes activation of neutrophils in whole blood. J Immunol 162(4): 2353-2357

17. Wang PY, Kao CH, Mui MY, Wang SJ (1993) Leukocyte infiltration in acute hemispheric ischemic stroke. Stroke 24(2): 236-240 